\section{Vertebral osteomyelitis presenting with pleuritic chest pain and bilateral pleural effusions}

\author{
P J Sullivan, D Currie, J V Collins, \\ D J Johnstone, A Morgan
}

\begin{abstract}
Pyogenic non-tuberculous vertebral osteomyelitis presented with pleuritic chest pain and basal shadowing on the chest radiograph.
\end{abstract}

Department of

Thoracic Medicine

PJ Sullivan

D Currie

J V Collins

Department of

Orthopaedic Surgery

D J Johnstone

Westminster Hospital, London SW1 2AP

Thanet District

General Hospital, Margate, Kent

CT9 4AN

A Morgan

Correspondence to: Dr P J Sullivan, Department of Thoracic Medicine, King's College School of Medicine and Dentistry, London SE5 9TG.

Accepted 28 November 1991

\begin{abstract}
Pyogenic non-tuberculous vertebral osteomyelitis may present with non-specific symptoms, which make diagnosis difficult. We describe a patient whose initial symptom was pleuritic chest wall pain associated with bilateral basal shadowing on the chest radiograph.
\end{abstract}

\section{Case report}

A 64 year old man presented with bilateral infrascapular pleuritic chest pain and breathlessness. He had had occasional night sweats but no other symptoms and previously he had been active and well. He had never smoked. On examination he was febrile $\left(37.6^{\circ} \mathrm{C}\right)$ and tachypnoeic with signs suggesting bilateral pleural effusions. There was no spine tender-

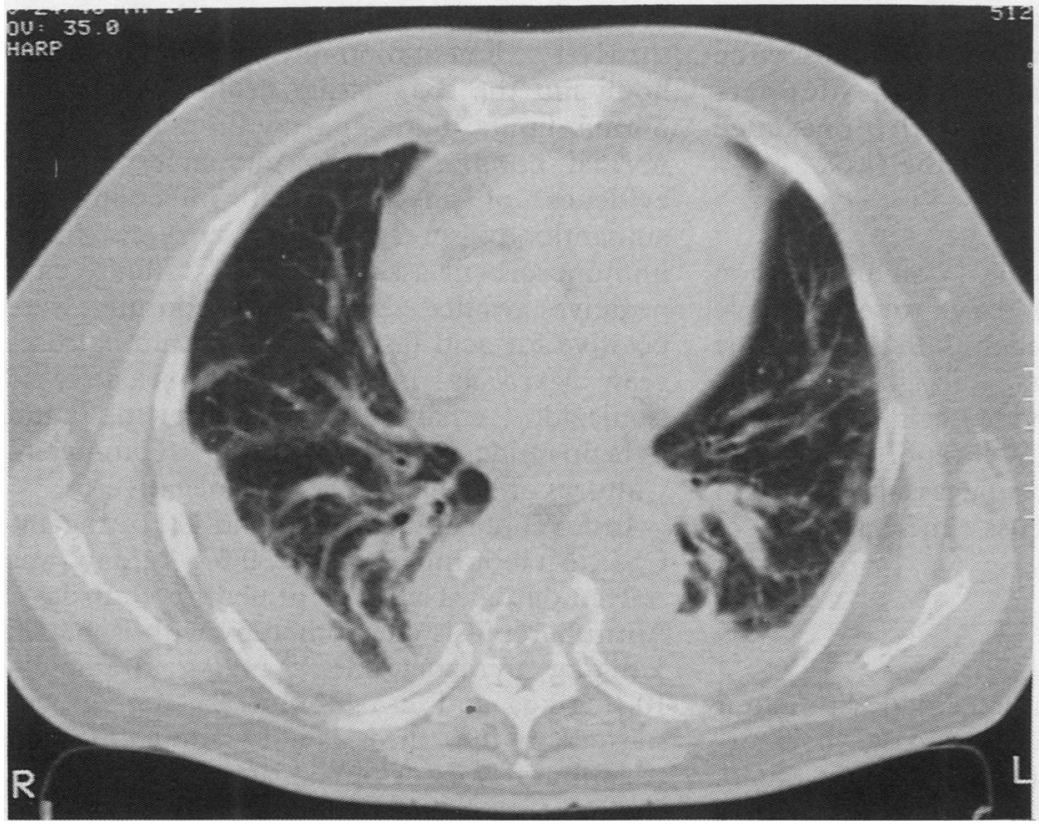

Computed tomogram of the thorax showing destruction of the $T 7$ vertebra and associated pleural changes. ness and straight leg raising was normal.

A chest radiograph showed bilateral basal pleural shadowing but the thoracic and lumbar spines were normal. Pleural aspiration was attempted but no fluid was obtained. He had microcytic anaemia (haemoglobin $(9 \mathrm{~g} / \mathrm{dl}$ ), an erythrocyte sedimentation rate of $117 \mathrm{~mm}$ in one hour, and a white cell count of $9 \times 10^{9} / 1$. Blood cultures were sterile. A ventilationperfusion scan showed matched defects.

With a presumptive diagnosis of pneumonia he was treated with intravenous benzylpenicillin and erythromycin and the radiographic appearances improved. After discharge he developed increasingly severe pain and required opiate analgesics. He became short of breath at rest and complained of frequent drenching night sweats. Chest radiographs then showed recurrent bilateral basal shadowing and the lateral film showed destruction and collapse of the body of the 7th thoracic vertebra with narrowing of the T7-8 disk space and sclerotic changes in the upper margin of T8. Computed tomography of the thorax showed degeneration of the trabecular pattern and loss of the cortical margin of the T7 vertebral body and bilateral pleural thickening. There was some basal atelectasis but the lungs were otherwise normal. Pleural aspiration under ultrasound control produced only $5 \mathrm{ml}$ of a bloodstained exudate, which contained numerous neutrophils and which grew no organisms. The pleura was thickened and a biopsy specimen consisted of fibrinous material and haemorrhagic granulation tissue. Osteomyelitis was suspected and costotransversectomy and vertebral bone biopsy were performed. Gelatinous material was removed. The histological appearances were those of acute osteomyelitis with no evidence of neoplasia. Staphylococcus aureus was cultured from the bone biopsy specimen. Culture for mycobacteria was negative. Blood cultures produced no growth.

The patient was treated with a prolonged course of flucloxacillin and fucidic acid and his pain resolved after six weeks.

\section{Discussion}

Non-tuberculous vertebral osteomyelitis may be a subacute disorder with an insidious onset. ${ }^{1}$ Two thirds of cases are not preceded by identifiable sepsis elsewhere ${ }^{2}$ and blood cultures are frequently negative. ${ }^{3}$ Radiographs of the spine may be normal at the time of presentation $^{4}$ and it may take up to eight weeks before radiological changes become apparent. ${ }^{5}$ The erythrocyte sedimentation rate is usually raised, often substantially, but the white cell count may remain within normal limits. ${ }^{6}$ There is often a delay in the diagnosis of vertebral osteomyelitis, partly because it is a disease of very low incidence in Britain ${ }^{7}$ and partly because the cardinal symptom, back pain, is common in the normal population.

This patient had no history of central back pain and the original symptoms and signs suggested a respiratory disorder. We suggest that the early symmetrical chest pains were 
caused by nerve root compression and that bilateral empyema developed as a consequence of direct spread from the infected vertebrae.

A literature search showed one previous report of pleural effusion associated with osteomyelitis. ${ }^{4}$ In that case the effusion was small and not associated with respiratory symptoms. This is the first reported case of prominent pleural pathological features associated with shortness of breath occurring as the presenting feature of pyogenic vertebral osteomyelitis.
1 Thompson D, Bannister P, Murphy P. Vertebral osteomyelitis in the elderly. $B M J 1988 ; 296: 1309-11$.

2 Silverthorne KG, Gillespie WJ. Pyogenic spinal osteomyelitis, a review of 61 cases. NZJMed 1986;99:62-5.

3 Osenbach RK, Hitcham PW, Menezes AH. Diagnosis and management of pyogenic vertebral osteomyelitis in adults. Surg Neurol 1990;33:266-75.

4 Carr AJ, Crow PJ. Vertebral osteomyelitis presenting with abdominal pain and a pleural effusion. $J R$ Coll Surg Edinb 1987;32:373.

5 Stone DB, Bonfiglia M. Pyogenic vertebral osteomyelitis; diagnostic pitfall for the internist. Arch Intern Med 1963; 112:491-500.

6 Fernandez-Ulloa M, Vasavada PJ, Hanslits ML, Volarich DT, Elgazzar AH, et al. Diagnosis of vertebral osteomyelitis: clinical, radiological and scintigraphic features. Orthopedics 1985;8:1144-50.

7 Digby KM, Kersley JB. Pyogenic non-tuberculous spinal infection. J Bone Joint Surg 1979;61:47-58.
Immune

thrombocytopenia complicating pulmonary tuberculosis: case report and investigation of mechanisms

\author{
Robert J Boots, Andrew W Roberts, \\ David McEvoy
}

Immune thrombocytopenia and pulmonary tuberculosis presented concurrently in a 20 year old Thai man as a bleeding diathesis. Intravenous immunoglobulin rapidly corrected the thrombocytopenia. Immunofiuorescence and immunoblot studies with platelets and mycobacteria showed the presence of platelet surface membrane IgG.

Isolated thrombocytopenia is an uncommon complication of pulmonary tuberculosis. ${ }^{12}$ The pathogenesis is believed to be immune destruction of platelets, though only one previous report has provided evidence to support this hypothesis. ${ }^{3}$ We report a case of immune thrombocytopenia associated with tuberculosis and discuss mechanisms of platelet sensitisation.

Thoracic Medicin R J Boots

D McEvoy

Department of

Haematology

A W Roberts

Royal Brisbane

Hospital, Herston,

Queensland, Australia 4029

Reprint requests to:

Dr R J Boots

Accepted 13 November 1991

\section{Case report}

A 20 year old Thai man presented with a four day history of recurrent mucosal haemorrhages and rectal bleeding with bright blood. He had been unwell for two weeks with a cough producing small amounts of purulent sputum, intermittent night sweats, fevers, and rigors. In the preceding four months he had noted lethargy and a $9 \mathrm{~kg}$ weight loss. There was no personal or family history of tuberculosis. A drug and toxin exposure history was non-contributory. He had emigrated from Thailand in 1980. A chest radiograph in 1982 had been reported as normal.

On examination he was febrile and weighed $42 \mathrm{~kg}$. Bilateral cervical lymphadenopathy was present, the glands ranging in size from 1 to $3 \mathrm{~cm}$. There was no hepatosplenomegaly. $\mathrm{He}$ had numerous cutaneous petechiae and haemorrhages in the oropharynx and there was bright blood on rectal examination. Scattered early inspiratory crackles were heard over the left upper zone of the chest.

A chest radiograph showed patchy left upper lobe and right upper and middle lobe opacities. The platelet count was 5 (normal range $140-400) \times 10^{9} / 1$; the white cell count was $12.7 \times 10^{9} / 1$ with a slight monocytosis. A microcytic anaemia of $11.7 \mathrm{~g} / 1$ with a mean corpuscular volume of $73.3 \mathrm{fl}$ was present. Haemoglobin E trait was noted. The erythrocyte sedimentation rate was $38 \mathrm{~mm}$ in one hour and the coagulation profile was within normal limits. Bone marrow biopsy showed a normal number of megakaryocytes and no evidence of mycobacterial infection. An autoantibody screen and an enzyme linked immunosorbent assay for HIV antibody gave negative results. Smears of sputum were positive for acid fast bacilli. Sputum cultures grew Mycobacterium tuberculosis sensitive to isoniazid, ethambutol, rifampicin, and pyrazinamide but resistant to streptomycin Cultures of bone marrow were negative.

Intravenous immunoglobulin $\mathbf{G}$ (IntragamCSL, Melbourne) infusion, $0.5 \mathrm{~g} \mathrm{~kg} /$ day, was started on day 1 and continued for four days. Antituberculosis chemotherapy with isoniazid $5 \mathrm{mg} / \mathrm{kg}$, pyrazinamide $35 \mathrm{mg} / \mathrm{kg}$, rifampicin $10 \mathrm{mg} / \mathrm{kg}$, and ethambutol $25 \mathrm{mg} / \mathrm{kg}$ was started on the third day. Pyrazinamide and ethambutol were stopped after two months. Owing to problems over compliance isoniazid and rifampicin were continued for nine months. 